\title{
¿Está justificado el clipaje aneurismático temprano en pacientes con hemorragia subaracnoidea de alto grado?
}

Ariel Varela Hernández¹, Félix Orellana Cortéz¹, Claudio Martínez Terreu ${ }^{1}$, Luis Lamus Aponte ${ }^{1}$, Patricio Herrera Astudillo ${ }^{1}$, Rodolfo Muñoz Gajardo', Reinaldo Torres Aravena1, Licenciada Cynthia Armijo Olivo'

1 Servicio de Neurocirugía. Hospital Regional de Talca. Maule, Chile.

Rev. Chil. Neurocirugía 43: 118-127, 2017

\section{Resumen}

Introducción: Aproximadamente el $40 \%$ de los pacientes que debutan con Hemorragia Subaracnoidea de causa aneurismática se presentan en pobre condición clínica, con mortalidad que se aproxima al 100\%. Las pautas de atención de estos enfermos son aun controvertidas. Dentro de dichas controversias resalta el tema de la utilidad o no del clipaje aneurismático temprano. En este artículo se analizan los resultados de una serie de pacientes operados. Material y Método: Se estudió una muestra no probabilística de los pacientes operados por el autor principal, con aneurismas intracraneales rotos en grado IV o V (WFNS), en las primeras 72 horas del debut, desde el 1 de enero de 2015 al 31 de diciembre de 2016, en el Hospital Regional de Talca, Maule. Se aplicaron técnicas de Chi cuadrado, ANOVA y regresión logística. Resultados: Se estudiaron 17 pacientes. La mortalidad general fue del $29,4 \%$ y el $41,2 \%$ se registró con buenos resultados de acuerdo a la escala de Rankin modificada. Los factores relacionados con la muerte o los malos resultados fueron el resangramiento asociado a hematoma intraparenquimatoso, el edema cerebral transoperatorio, la gradación alta en la escala de Fisher y la no realización de ventriculostomía externa antes de la oclusión del aneurisma. Conclusiones: El clipaje aneurismático es factible de realizarse en estos pacientes con pobre condición clínica, sin embargo, un alto número persiste con discapacidad severa. Se requieren estudios que comprueben la utilidad de estrategias más efectivas para la selección de los pacientes a operar.

Palabras clave: Aneurisma intracraneal roto, hemorragia subaracnoidea aneurismática, cirugía, pronostico.

\begin{abstract}
Introduction: Approximately $40 \%$ of patients with Subarachnoid Hemorrhage due to aneurysmal rupture are presented in poor clinical condition, with a mortality approaching $100 \%$. The management of these patients are still controversial. Among these controversies, highlights the issue of the utility or not of early aneurysmal clipping. In this article we analyze the results of a series of patients operated on. Material and Method: We studied a non-probabilistic sample of patients operated by the main author, with ruptured intracranial aneurysms in grade IV or V (WFNS), in the first 72 hours of the debut, from January 1 , 2015 to 31 December 2016, at the Regional Hospital of Talca, Maule. Chi square, ANOVA and logistic regression techniques were applied. Results: We studied 17 patients. Overall mortality was $29.4 \%$ and $41.2 \%$ was recorded with good results according to the modified Rankin scale. Factors related to death or poor results were rebleeding associated with intraparenchymal hematoma, transoperative cerebral edema, high gradation on Fisher scale and no external ventriculostomy performed before aneurysm occlusion. Conclusions: Aneurysmal clipping is feasible in these patients with poor clinical condition, however a high number persists with severe disability. Studies that prove the utility of more effective strategies for the selection of patients to be operated are required.
\end{abstract}

Key words: Rupture intracranial aneurysm, aneurysmal subarachnoid hemorrhage, surgery, prognosis. 


\section{Introducción}

La hemorragia subaracnoidea aneurismática (HSAa) constituye una entidad de gran relevancia en la medicina contemporánea. La misma representa aproximadamente el $10 \%$ de todos los casos detectados con enfermedades cerebrovasculares y se detecta fundamentalmente en personas con edades sociales activas ${ }^{25}$.

A pesar de los tremendos avances introducidos en la práctica clínica en las últimas décadas; encaminadas a un diagnóstico más oportuno, atención neurointensiva y técnicas de tratamiento de menor invasividad; la historia natural de la enfermedad se mantiene con gran agresividad y elevados índices de morbilidad y mortalidad ${ }^{23,26}$.

Dentro de este conjunto de enfermos, un subgrupo que representa aproximadamente el $40 \%$ de los mismos, ubicado en el extremo más crítico del diapasón de las escalas clínicas de HSAa vigentes (grados IV o V), tienen una situación aún más comprometida y sombría. La utilidad del clipaje aneurismático temprano (en las primeras 72 horas del debut de la hemorragia) en los mismos, ha sido puesto en duda por muchos autores con las fundamentaciones de existencia de lesión neurológica catastrófica y la complejización técnica de la exposición y clipaje aneurismáticos. Además, el desarrollo de las técnicas endovasculares, que evitan la manipulación del encéfalo, motiva que sean preferidas por muchos en estas circunstancias ${ }^{4}$.

De manera contraria, la posición de la neurocirugía contemporánea se inclina cada vez más a conductas agresivas con estos pacientes que garanticen el aislamiento del aneurisma de la circulación sistémica antes de producirse el resangramiento, el lavado del espacio subaracnoideo de sustancias espasminógenas; así como el control de la hipertensión endocraneana mediante la evacuación de hematomas, drenaje de líquido cefalorraquídeo (LCR) o craniectomías descompresivas. No obstante, un número aún muy elevado de los mismos fallecen o se mantienen con secuelas muy invalidantes, elevando ostensiblemente el costo social ${ }^{31}$. La selección del momento de realización de la cirugía en estas condiciones se mantiene como un tema álgido y no existen estándares aceptados a nivel mundial $^{12}$. En opinión del autor el clipa- je aneurismático temprano en pacientes con HSAa de alto grado estaría justificado sobre bases selectivas, por lo que se requieren aún de estudios que demuestren herramientas predictivas efectivas para dirigir de forma más racional estas conductas.

Para fundamentar esta idea se realizó este trabajo que persigue mostrar los resultados de una serie de pacientes con HSAa operados en grados IV o V, en cuanto a la factibilidad de la cirugía para ocluir el aneurisma, la aparición de complicaciones, el resultado final del proceso de atención del paciente y la determinación de factores de mal pronóstico.

\section{Material y Método}

Diseño y contexto: Se realizó un estudio observacional, retrospectivo, transversal y analítico con los pacientes atendidos con HSAa en grado IV o V según la clasificación de la Federación Mundial de Cirujanos Neurológicos (WFNS), a los que se le practicó oclusión microquirúrgica del aneurisma en las primeras 72 horas del debut del ictus, en el Hospital Regional de Talca, Maule, desde el primero de enero de 2015 al 31 de diciembre de 2016.

Muestra: Se creó una muestra no probabilística usando los criterios siguientes:

Criterios de inclusión: Pacientes mayores de 18 años de edad, pacientes detectados con HSAa grado IV o V según escala de la WFNS (Anexo 1) en el momento de la decisión de la oclusión microquirúrgica del aneurisma, pacientes en los que dicho procedimiento neuroquirúrgico se realizó en las 72 horas subsiguientes al debut del ictus en el Hospital Regional de Talca por el autor, acompañado por un equipo multidisciplinario.

Criterios de exclusión: Pacientes fallecidos antes de arribar al citado centro hospitalario o durante la reanimación en el servicio de urgencias del mismo, pacientes con aneurismas rotos asociados a otras lesiones vasculares estructurales intracraneales (Ejemplo Malformación arterio venosa, etcétera), pacientes con aneurismas de probable causa infecciosa o traumática, pacientes excluidos de la cirugía; lo cual estuvo relacionado con estado moribundo, expectativa de vida inferior a 6 meses, Glasgow postreanimación inferior a 6 puntos o respuesta motora inferior a 4 puntos en dicha escala, lesiones encefálicas isquémicas relevantes en la tomografía computarizada (TC) de cráneo o no aporte del consentimiento informado por los familiares, así como los pacientes derivados a otros centros hospitalarios para su solución quirúrgica.

Recolección de la información: Se revisaron las declaraciones de enfermedad AUGE con el diagnóstico de HSAa en el período de tiempo y centro hospitalario antes referidos. Las fichas clínicas de cada uno de ellos, incluyendo los informes imagenológicos y los protocolos operatorios, fueron revisadas por el autor principal. En aquellos casos en que parte de la evolución posterior a la cirugía se llevó a cabo en otro centro hospitalario o que no presentaron consultas de control en los últimos 3 meses, se contactó al paciente o familiares de forma presencial o telefónica. Con los datos obtenidos se completó un cuestionario confeccionado al efecto.

Proceso de atención hospitalaria: En los pacientes estudiados se siguieron las recomendaciones de la Guias GES vigentes en Chile $^{34}$ para la atención de los pacientes con HSAa y que en relación al presente estudio se sistematizaron de la siguiente forma:

Período pre oclusión del aneurisma: Incluyó la reanimación cardioventila-
Anexo 1. Escala de HSA de Federación Mundial de Cirujanos Neurológicos (WFNS)

\begin{tabular}{|c|c|c|}
\hline Grado & Glasgow & Defecto motor \\
\hline I & 15 & No \\
\hline II & $14-13$ & No \\
\hline III & $14-13$ & $\mathrm{Si}$ \\
\hline IV & $12-7$ & $\mathrm{Si} / \mathrm{No}$ \\
\hline V & $3-6$ & $\mathrm{Si} /$ No \\
\hline
\end{tabular}


toria cuando fue requerida, intubación endotraqueal en pacientes con escala de Glasgow menor a 9 puntos, disritmia ventilatoria o hipoxemia, diagnóstico de la HSA con TC (Equipo GE healthcare /16 canales/light speed) de cráneo simple, urgente. Hospitalización en servicio de paciente crítico, tratamiento farmacológico con fluidoterapia, analgésicos, control de la presión arterial sistólica entre 120 y $159 \mathrm{~mm} \mathrm{Hg}$, nimodipina $60 \mathrm{mg}$ V.o c/4 h, Fenitoína I.V o V.O o Levetiracetam V.O y antiácidos; mantención del tratamiento con estatinas en los casos con indicación previa. Diagnóstico de aneurisma intracraneal roto mediante Angio TC cerebral urgente (Equipo GE healthcare /16 canales/light speed). Se realizó ventriculostomía al exterior urgente en los casos con hidrocefalia aguda o para el monitoreo continuo de la presión intracraneal (PIC). En los casos con hematoma intraparenquimatosos cerebrales se realizó la evacuación de los mismos durante la cirugía para la oclusión del aneurisma.

Oclusión del aneurisma: Se escogieron abordajes fronto latero pterionales o paramedianos interhemisféricos de acuerdo a la localización del aneurisma roto. En todos los casos se excluyó el aneurisma mediante clipaje con el apoyo del microscopio quirúrgico (Carl Zeiss S88). También se realizó evacuación de hematomas cuando correspondió y el lavado abundante del espacio subaracnoideo con suero isotónico estéril en todos los casos. La realización de ventriculostomía externa durante el proceder se llevó a cabo en algunos pacientes cuando fue necesario para favorecer la relajación cerebral. El empleo de clipaje arterial transitorio y la apertura de la lámina terminalis se realizó de acuerdo a la decisión del cirujano en cada caso en particular.

Período post oclusión del aneurisma: Se realizó en servicio de atención a pacientes críticos. Se mantuvo en todos los casos al paciente sedado, con ventilación mecánica durante un período de tiempo determinado por la evolución de cada caso. Se aplicó hipertensión euvolémica ligera (presión arterial media de 100 a $110 \mathrm{~mm} \mathrm{Hg}$ ) y Nimodipina $60 \mathrm{mg} \mathrm{c} / 4 \mathrm{~h}$ por vía enteral hasta cumplir 21 días de tratamiento. En los casos con ventriculostomía externa se mantuvo el monitoreo continuo de la PIC, además se realizó el monitoreo con índice biespectral e imagenológico estructural con TC y Angio TC ce- rebrales. En los casos complicados con Defecto Isquémico Tardío (DIT) se aumentó el umbral de hipertensión euvolémica y en las lesiones isquémicas con efecto de masa se procedió con la craniectomía descompresiva. A pesar de la consideración en algunos casos para Angioplastia Transluminar, la misma no pudo realizarse en ninguno de estos casos ya que el estado clínico impidió el traslado a otros centros distantes para efectuar dicho tratamiento. Se efectuó profilaxis farmacológica de la trombosis venosa profunda con Clexane $40 \mathrm{mg}$ s.c diarios después de 24 horas, con el aval de la TC de cráneo simple. Se aplicaron continuamente técnicas de neurorehabilitación en correspondencia al período evolutivo de los pacientes.

Operacionalización de variables: En el cuestionario diseñado se incluyeron las siguientes variables, a las cuales se le aplicó un proceso de codificación, donde el mayor valor se correspondió con la mayor gravedad del evento: edad (según grupos de edades: 18-50 años $=1,51-70$ años $=2$, mayor a 70 años $=3$ ), sexo (masculino $=1$, femenino $=2$ ), comorbilidad (sin comorbilidad $=1$, comorbilidad sin hipertensión arterial $(\mathrm{HTA})=2, \mathrm{HTA}=3$, HTA asociada a Diabetes Mellitus $(\mathrm{DM})=4$, múltiples asociadas a HTA = 5), síntomas de aviso ( in síntomas de aviso $=1$, síntomas adecuadamente reconocidos $=2$, síntomas no reconocidos $=3$ ), escala de Glasgow (valor detectado en el último examen clínico antes de la oclusión de aneurisma: $12-9$ puntos $=1$, menor a 9 puntos $=2$ ), escala de la WFNS (valor detectado en el momento de la decisión de la oclusión del aneurisma: IV $=1, V=2$ ), escala de Fisher modificada (Anexo 2) (según el último examen tomográfico antes de la oclusión del aneurisma: II = 1 , III = 2 , IV = 3), locali- zación del aneurisma roto (origen de la comunicante posterior $=1$, bifurcación de la carótida interna $=2$, bifurcación de la cerebral media $=3$, pericalloso $=$ 4 , comunicante anterior $=5$ ), tamaño aneurismático $(0-5 \mathrm{~mm}=1,6-10 \mathrm{~mm}=$ 2, 11-24 $\mathrm{mm}=3$ ), multiplicidad aneurismática ( $\mathrm{no}=1$, si $=2$ ), complicaciones intracraneales previa a la oclusión (sin complicaciones $=1$, convulsión $=2$, hidrocefalia $=3$, hematoma intraparenquimatoso $(\mathrm{HIP})=4$, resangramiento $=$ 5 , resangramiento asociado a HIP $=6$ ), complicaciones extracraneales previa a la oclusión (sin complicaciones $=1$, broncoaspiración $=2$, disritmia ventilatoria $=3$, paro cardioventilatorio $=4$ ), realización de ventriculostomía externa (no $=1$, preoperatoria $=2$, transoperatoria $=3$, postoperatoria $=4$ ), momento de la cirugía (primeras 24 horas del debut de la HSA = 1, 25-48 horas $=2$, 49-72 horas $=3$ ), tipo de clipaje (simple $=1$, múltiple $=2$ ), necesidad de reposicionamiento del clip (durante el propio acto quirúrgico: no $=1$, si $=2$ ), empleo de clipaje transitorio del vaso madre (no $=1$, menor o igual a 5 minutos $=2$, $6-15$ minutos $=3$, en dos momentos $=$ 4), apertura de la lámina terminalis (si $=1$, no $=2$ ), complicaciones transoperatorias ( $\sin$ complicaciones $=1$, edema cerebral $=2$, rotura aneurismática $=3$ ), complicaciones postoperatorias ( $\sin$ complicaciones $=1$, neumonía asociada a ventilación $=2$, hidrocefalia transitoria $=3$, diabetes insípida/natriuresis cerebral $=4$, ventriculitis $=5$, vasoespasmo sintomático $=6$, shock $=$ 7), momento en que se registraron los resultados (durante el ingreso $=1$, del alta hospitalaria a los 2 meses $=2,3-12$ meses $=3,13-24$ meses $=4$ ), escala de Rankin modificada (Anexo 3) (I-III (buenos resultados) $=1, \mathrm{IV}-\mathrm{VI}$ (malos resultados $)=2$, mortalidad (vivo $=1$, muerte tardía (después del alta hospi-

Anexo 2.

Escala de Fisher modificada

1 Hemorragia subaracnoidea fina (menor a $1 \mathrm{~mm}$ ), sin hemorragia intraventricular

2 Hemorragia subaracnoidea fina (menor a $1 \mathrm{~mm}$ ), con hemorragia intraventricular

3 Hemorragia subaracnoidea gruesa (mayor o igual a $1 \mathrm{~mm}$ ), sin hemorragia intraventricular

$4 \quad$ Hemorragia subaracnoidea gruesa (mayor o igual a $1 \mathrm{~mm}$ ), con hemorragia intraventricular 
talario o traslado al hospital de base) $=2$, temprana (durante la hospitalización) = 3).

Procedimientos estadísticos: Se utilizó el programa S.P.S.S v 18.0, usando técnicas de estadística descriptiva y comprobatorias para variables no paramétricas (Chi cuadrado, ANOVA y regresión logística binaria), empleando un intervalo de confianza del $95 \%$.

Consideraciones éticas: La investigación fue avalada por el servicio de Neurocirugía y el Comité de Ética del Hospital Regional de Talca. Las decisiones terapéuticas empleadas se correspondieron con las recomendadas en la literatura universal ${ }^{8,43}$ y las Guías GES vigentes en Chile; en todo caso las mismas se aplicaron de forma consensuada en el servicio de Neurocirugía del Hospital Regional de Talca y con el aval del consentimiento informado del tutor legal del paciente. Los actos quirúrgicos se llevaron a cabo por al menos dos Neurocirujanos y un anestesiólogo con experiencia en procederes neuroanestésicos. La atención pre y postoperatoria se llevó a cabo en Unidades de Pacientes Críticos. Los datos obtenidos a partir de la fichas de los pacientes se mantuvieron en confidensiabilidad en todo momento.

\section{Resultados}

La muestra estuvo integrada por 17 enfermos, que representaron el $43,5 \%$ del universo de 39 pacientes operados de forma temprana por aneurismas intracraneales rotos por el autor, en el período de tiempo estudiado. Se encuestaron pacientes entre 18 y 82 años, la media de la serie fue de 56,1 años. Predominó el sexo femenino con 12 enfermos (70,6\%). La presentación con grado $\mathrm{V}$ de la WFNS se detectó en 10 pacientes $(58,8 \%)$ y con grado IV de Fisher en 14 de los mismos $(82,4 \%)$. Los pacientes estudiados presentaron aneurismas de la circulación anterior, predominaron los originados en el segmento comunicante posterior de la arteria carótida interna con 6 (35,3\%) y en la bifurcación de la arteria cerebral media en $5(29,4 \%)$. En 9 pacientes $(52,9 \%)$ se detectaron aneurismas pequeños y en $7(41,2 \%)$ fueron grandes. Todos los enfermos fueron operados en las primeras 72 horas del debut de la HSAa, en $3(17,6 \%)$ la oclusión del aneurisma se realizó en las primeras 24 horas. En 12 enfermos (70,5\%) se practicó ventriculostomía al exterior, en $7(41,2 \%)$ de los mismos previa a la craneotomía, en $3(17,6 \%)$ durante el abor-

\section{Anexo 3.}

Escala de Rankin modificada

\begin{tabular}{|l|l|}
\hline 0 & Sin síntomas ni limitaciones \\
\hline 1 & $\begin{array}{l}\text { No discapacidad significativa: algunos síntomas pero sin limitaciones para } \\
\text { las actividades cotidianas y laborales }\end{array}$ \\
\hline 2 & $\begin{array}{l}\text { Discapacidad leve: algunas limitaciones para sus actividades laborales y } \\
\text { cotidianas, se mantiene independiente para las actividades básicas de la } \\
\text { vida cotidiana }\end{array}$ \\
\hline 3 & $\begin{array}{l}\text { Discapacidad moderada: satisface sin ayuda las actividades individuales. } \\
\text { Ejemplo: alimentación, aseo, etcétera }\end{array}$ \\
\hline 4 & $\begin{array}{l}\text { Discapacidad moderada - severa: necesita asistencia para las actividades } \\
\text { básicas, pero de forma continua }\end{array}$ \\
\hline 5 & Discapacidad severa: totalmente dependiente, requiere asistencia continua \\
\hline 6 & Muerte \\
\hline
\end{tabular}

\begin{tabular}{|c|c|}
\hline Características & Valores \\
\hline Edad & $\begin{array}{l}\text { Máximo: } 82 \text { años } \\
\text { Mínimo: } 18 \text { años } \\
\text { Media: } 56,1 \text { años }\end{array}$ \\
\hline Sexo & $\begin{array}{l}\text { Femenino: } 12(70,6 \%) \\
\text { Masculino: } 5(29,4 \%)\end{array}$ \\
\hline Escala de la WFNS & $\begin{array}{l}\text { IV: } 10(58,8 \%) \\
\text { V: } 7(41,2 \%)\end{array}$ \\
\hline Escala de Fisher & $\begin{array}{l}\text { IV: } 14(82,4 \%) \\
\text { III: } 3(17,6 \%)\end{array}$ \\
\hline Localización aneurismática & $\begin{array}{l}\text { Origen comunicante posterior: } 6(35,3 \%) \\
\text { Bifurcación cerebral media: } 5(29,4 \%) \\
\text { Bifurcación carótida interna: } 2(11,8 \%) \\
\text { Comunicante anterior: } 2(11,8 \%) \\
\text { Pericallosa: } 2(11,8 \%)\end{array}$ \\
\hline Diámetro aneurismático & $\begin{array}{l}\text { Pequeño: } 9(52,9 \%) \\
\text { Mediano: } 7(41,2 \%) \\
\text { Grande: } 1(5,9 \%)\end{array}$ \\
\hline Momento de la cirugía & $\begin{array}{l}\text { 49-72 horas: } 7(41,2 \%) \\
\text { 25-48 horas: } 7(41,2 \%) \\
\text { Menos } 24 \text { horas: } 3(17,6 \%)\end{array}$ \\
\hline $\begin{array}{l}\text { Realización de ventriculostomía al } \\
\text { exterior }\end{array}$ & $\begin{array}{l}\text { Preoperatorio: } 7(41,2 \%) \\
\text { Transoperatorio: } 3(17,6 \%) \\
\text { Postoperatorio: } 2(11,8 \%) \\
\text { Total: } 12(70,5 \%) \\
\end{array}$ \\
\hline Momento de la encuesta & $\begin{array}{l}\text { Máximo: } 24 \text { meses } \\
\text { Mínimo: } 7 \text { días } \\
\text { Media: } 9,9 \text { meses }\end{array}$ \\
\hline
\end{tabular}

daje del aneurisma y en $2(11,8 \%)$ en el período postoperatorio. Los pacientes fueron encuestados entre 7 días y 24 meses con una media de 9,9 meses, los valores más bajos se registraron en los enfermos fallecidos. (Tabla 1). 
Dentro de las complicaciones preoperatorias (se refiere a la cirugía para oclusión del aneurisma), la hidrocefalia se detectó en 3 pacientes (17,6\%); el hematoma intraparenquimatoso, el resangramiento y la asociación de estos dos últimos, se registró en 2 pacientes $(11,8 \%)$ en cada caso. Durante el desarrollo de la cirugía se presentó el edema cerebral y la ruptura aneurismática en 3 operados $(17,6 \%)$ de manera respectiva. En el período postoperatorio se detectó la neumonía asociada a la ventilación mecánica prolongada en 8 enfermos $(47,1 \%)$, el vasoespasmo sintomático en $6(35,3 \%)$ y en 1 enfermo $(5,9 \%)$ la ventriculitis. (Tabla 2$)$.

Se detectaron 5 pacientes $(29,4 \%)$ fallecidos, en 3 de los mismos (17,6\%) la muerte ocurrió varias semanas después del alta hospitalaria y en 2 $(11,8 \%)$ durante el propio período de hospitalización. Se registraron buenos resultados en la escala de Rankin modificada en 7 (41,2\%). Dentro del grupo de los pacientes con malos resultados se destacan 2 que en la actualidad muestran mejoría progresiva con la rehabilitación. (Figura 1).

En las pruebas estadísticas bivariadas se encontró relación del resangramiento con hematoma intraparenquimatoso en el periodo preoclusión, del edema cerebral transoperatorio, la escala de Fisher y la realización de ventriculostomía externa, con la mortalidad. En el caso de las últimas dos variables citadas, se encontró también relación con los malos resultados medidos según la escala de Rankin modificada. (Tabla 3). En el análisis de varianza se observó que el mayor valor de las medias cuadráticas de la mortalidad y los malos resultados medidos con la escala de Rankin modificada, se relacionaron en primer lugar con la realización transoperatoria de la ventriculostomía externa y en segundo lugar cuando esta se practicó posterior a la oclusión quirúrgica del aneurisma. (Figura 2).

El modelo de predicción de la mortalidad en esta serie, obtenido a partir de la regresión logística binaria, identificó la ocurrencia de edema cerebral transoperatorio como la variable más significativa. El pronóstico de predicción correcta de dicho modelo fue del $88,2 \%$ y el O.R de 59,1. (Tabla 4).

El resultado de la aplicación de la regresión logística binomial mostró un pronóstico de los malos resultados, según la escala de Rankin modifica-

\begin{tabular}{|c|c|}
\hline Complicaciones & Valores \\
\hline Preoperatorias & $\begin{array}{l}\text { Hidrocefalia aguda: } 3(17,6 \%) \\
\text { Hematoma intraparenquimatoso: } 2(11,8 \%) \\
\text { Resangramiento: } 2(11,8 \%) \\
\text { Resangramiento con hematoma intraparenquimatoso: } 2 \\
(11,8 \%)\end{array}$ \\
\hline Transoperatorias & $\begin{array}{l}\text { Edema cerebral: } 3(17,6 \%) \\
\text { Rotura aneurismática: } 3(17,6 \%)\end{array}$ \\
\hline Postoperatorias & $\begin{array}{l}\text { Neumonía asociada a la ventilación: } 8(47,1 \%) \\
\text { Vasoespasmo sintomático: } 6(35,3 \%) \\
\text { Ventriculitis: } 1(5,9 \%)\end{array}$ \\
\hline
\end{tabular}

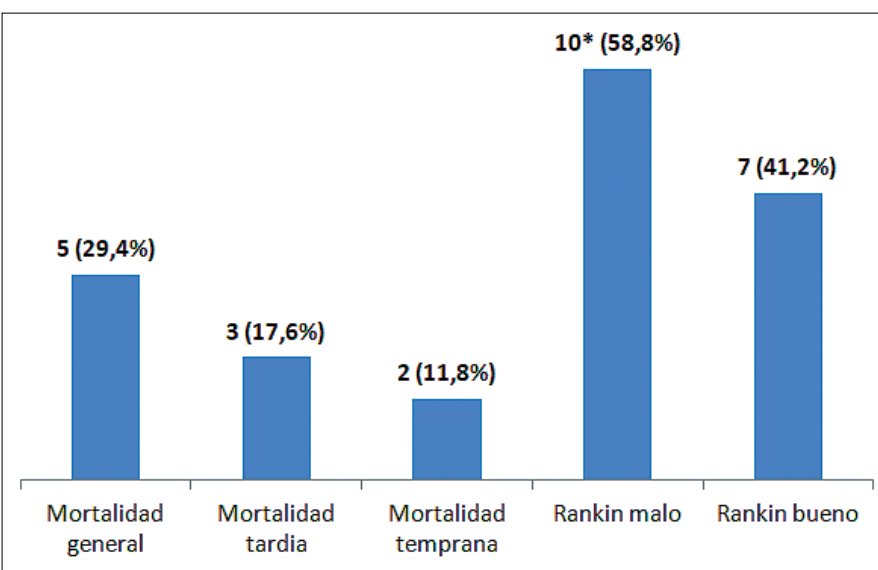

Figura 1. Pacientes según resultados. *Incluyen 2 pacientes con 4 meses de seguimiento que en la actualidad muestran mejoría progresiva de su estado funcional con la neurorehabilitación.

Tabla 3.

Resumen de variables relacionadas con la mortalidad. ( $\chi^{2}$ y ANOVA)

\begin{tabular}{|l|l|l|}
\hline Variables & Valor de $\mathbf{p}$ & $\mathbf{I . C}$ 95\% \\
\hline $\begin{array}{l}\text { Resangramiento con hematoma intrapa- } \\
\text { renquimatoso }\end{array}$ & 0,018 & $1,05-1,54$ \\
\hline Edema cerebral transoperatorio & 0,001 & $1,33-1,85$ \\
\hline Escala de Fisher* & 0,02 & $1,05-1,54$ \\
\hline Realización de venticulostomía externa** $^{*}$ & 0,012 & $0,31-1,41$ \\
\hline
\end{tabular}

*También relacionada con malos resultados en la escala de Rankin ( $p=0,02 ;$ I.C 95\%: 1,33 - 1,85); ${ }^{\star *}$ También relacionada con malos resultados en la escala de Rankin ( $p=0,28$; I.C 95\%: 0,35 - 1,21).

da, en esta serie del $88,2 \%$ cuando se presentó elevación de la gradación en la escala de Fisher y la ocurrencia de complicaciones postoperatorias, con un O.R de 69,1. (Tabla 5).

\section{Discusión}

Características generales de la serie: La media de edad de la serie presenta$\mathrm{da}$, el predominio del sexo femenino y 


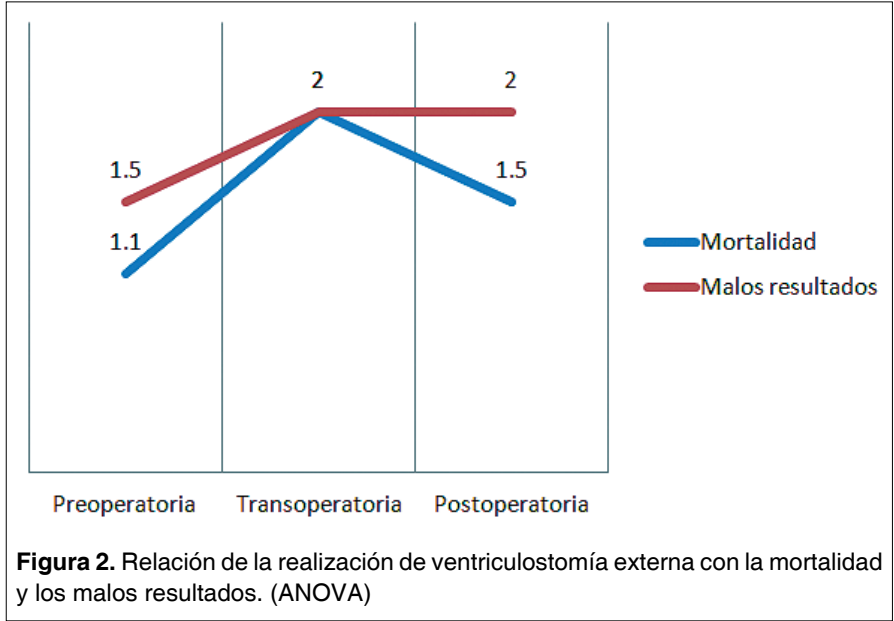

Tabla 4.

Modelo de predicción de la mortalidad

\begin{tabular}{|c|c|c|c|c|c|c|}
\hline \multicolumn{2}{|c|}{ Observado } & \multicolumn{3}{|c|}{ Pronosticado } & \multirow{3}{*}{$\begin{array}{l}\begin{array}{l}\text { Signifi- } \\
\text { cación al } \\
\text { cambio }\end{array} \\
0,01\end{array}$} & \multirow{3}{*}{$\begin{array}{r}\text { O.R } \\
\\
59,1\end{array}$} \\
\hline & & $\begin{array}{l}\text { No } \\
\text { Fallecidos }\end{array}$ & Fallecidos & $\begin{array}{l}\% \\
\text { correcto }\end{array}$ & & \\
\hline \multirow{2}{*}{$\begin{array}{l}\text { Edema } \\
\text { cerebral } \\
\text { transope- } \\
\text { ratorio }\end{array}$} & $\begin{array}{l}\text { No } \\
\text { Fallecidos }\end{array}$ & 12 & 0 & 100 & & \\
\hline & Fallecidos & 2 & 3 & 60 & & \\
\hline \multicolumn{4}{|l|}{$\%$ global } & 88,2 & & \\
\hline
\end{tabular}

\begin{tabular}{|c|c|c|c|c|c|c|}
\hline \multicolumn{2}{|c|}{ Observado } & \multicolumn{3}{|c|}{ Pronosticado } & \multirow{3}{*}{\begin{tabular}{|c|}
$\begin{array}{l}\text { Signifi- } \\
\text { cación al } \\
\text { cambio }\end{array}$ \\
0,01
\end{tabular}} & \multirow{3}{*}{$\begin{array}{r}\text { O.R } \\
\\
69,1 \\
\end{array}$} \\
\hline & & $\begin{array}{l}\text { Rankin } \\
\text { I - III }\end{array}$ & \begin{tabular}{|l} 
Rankin \\
IV - VI
\end{tabular} & $\begin{array}{l}\% \\
\text { correcto }\end{array}$ & & \\
\hline \multirow{2}{*}{$\begin{array}{l}\text { Escala } \\
\text { de Fisher } \\
\text { Compli- } \\
\text { caciones } \\
\text { postope- } \\
\text { ratorias }\end{array}$} & $\begin{array}{l}\text { Rankin } \\
\text { I - III }\end{array}$ & 5 & 2 & 71,4 & & \\
\hline & $\begin{array}{l}\text { Rankin } \\
\text { IV - VI }\end{array}$ & 0 & 10 & 100 & & \\
\hline \multicolumn{4}{|l|}{$\%$ global } & 88,2 & & \\
\hline
\end{tabular}

de aneurismas pequeños, se corresponden con lo publicado en la literatu$\mathrm{ra}^{10,21}$. Llama la atención la indicación quirúrgica en pacientes octogenarios; los resultados alcanzados hoy en día en este tipo de cirugía, conjugados con el envejecimiento poblacional a nivel mundial, han provocado que en relación a la indicación quirúrgica se preste más importancia al estado funcional del paciente y a su expectativa de vida que a su edad cronológica ${ }^{13}$.

Se operaron sólo aneurismas de la circulación anterior y dentro de ellos predominaron los del origen de la arteria comunicante posterior y cerebral media, los que junto con los de la arteria comunicante anterior son los más reportados en trabajos similares ${ }^{3,5}$. De forma general en el servicio neuroquirúrgico donde se desarrolló la investigación, los aneurismas de la circulación posterior son referidos a otros centros para tratamiento endovascular. La ventriculostomía al exterior, si bien fue ampliamente usada, no se aplicó en todos los enfermos. En la actualidad esta medida se considera de gran utilidad con el objetivo de aliviar la hipertensión endocraneana inicial y mejorar el estado clínico de estos enfermos. Aunque teóricamente la misma pudiera favorecer el resangramiento por aumento de la presión transmural a nivel del aneurisma, los diferentes estudios publicados la han mostrado como una medida ventajosa en la atención de estos pacientes ${ }^{7}$.

En todos los pacientes la cirugía para la oclusión del aneurisma se efectuó dentro de las primeras 72 horas del debut, hecho demostrado como la medida de mayor utilidad en la profilaxis del resangramiento aneurismático; además de ofrecer seguridad para el empleo de tratamientos para la profilaxis o control del defecto neurológico isquémico tardío $^{20}$. Oudshoorn y colaboradores ${ }^{27}$ plantean la utilidad de la cirugía ultratemprana (en las primeras 24 horas) bajo el fundamento de que el momento de mayor riesgo de resangramiento es en las primeras 24 horas, sin embargo, aún no llega a demostrarse totalmente las ventajas de esta postura en relación a la cirugía en las primeras 72 horas del debut. El tiempo de evolución medio al momento de la investigación de aproximadamente 10 meses, es favorable para estimar de manera más real los resultados que se muestran.

Análisis de las complicaciones y los resultados: En el período preoclusión del aneurisma se encontraron tres complicaciones; la hidrocefalia aguda, el hematoma intraparenquimatoso y el resangramiento. De hecho uno de los aspectos de mayor peso en la definición del mal pronóstico de estos pacientes, es precisamente la mayor incidencia en los mismos de estas graves 
complicaciones y otras también muy relevantes como la disfunción pulmonar y cardíaca ${ }^{18,22,41}$.

La ruptura aneurismática transoperatoria ocurrió, en los tres pacientes reportados, durante el proceso de disección del aneurisma, en todos fue posible en control de la complicación mediante el empleo de clipaje transitorio del vaso madre, finalización de la disección e instalación del o los clips definitivos. En los casos donde a pesar del drenaje de LCR y el uso de agentes osmolares no se logró una adecuada relajación cerebral antes de iniciar el cierre quirúrgico, se optó por no reponer el flap craneal, ampliar los límites de craneotomía y el empleo de duroplastia expansiva.

En el posoperatorio se presentó un solo caso de ventriculitis, el cual pudo ser resuelto con tratamiento antimicrobiano sistémico e intraventricular. Predominó la presentación de vasosespasmo sintomático, temible complicación también más frecuente en los enfermos con HSAa de alto grado. A pesar del arsenal de técnicas de neuromonitoreo y terapéutico vigente, esta complicación es la de mayor mortalidad y morbilidad en los enfermos con aneurismas ya excluidos y se mantiene como un reto por saldar aun en la atención neurointensiva de los enfermos con HSAa ${ }^{1,2,11,17,35}$

Es bien reconocido que hasta un $40 \%$ de los pacientes con HSAa se presentan con alto grado y que los resultados generales son aun desfavorables en más del $60 \%$ de los mismos cuando se aplica una atención neuroquirúrgica intensiva, en los que no, la mortalidad supera el $90 \%$, debido a resangramiento en la mayoría de los $\operatorname{casos}^{6,44,46,47}$. Los resultados de esta serie en cuanto a mortalidad y grado de discapacidad, medidos con la escala de Rankin modificada, son comparables con lo reportado en la actualidad. Zeng y colaboradores ${ }^{45}$ contrastan en su estudio una mortalidad quirúrgica del $46 \%$ con una del $80 \%$ del grupo de pacientes no operados. Pan y colaboradores ${ }^{28}$ reportaron una mortalidad del $22,2 \%$ y buenos resultados en el $44,4 \%$.

A pesar de que las técnicas endovasculares son preferidas por muchos para la oclusión de los aneurismas rotos en los pacientes con HSAa de alto grado, al evitar la manipulación de un cerebro hostil para la adecuada exposición y tratamiento de la lesión ${ }^{19}$, los resultados que presentamos con la cirugía a cielo abierto son comparables con los de Goel y colaboradores ${ }^{14}$ con mortalidad del $29,1 \%$, malos resultados en el $41,7 \%$ y buenos resultados en el $29,2 \%$.

En esta serie se empleó la cirugía a cielo abierto en todos los casos, en ninguno de ellos las complicaciones surgidas en el trasoperatorio impidieron que se cumpliera el objetivo de la cirugía por lo que puede asegurarse que con el empleo del drenaje de LCR de las cisternas basales y del sistema ventricular, así como la aplicación de los principios de la neuroanestesia, puede lograrse exponer y excluir el aneurisma roto en la gran mayoría de los casos, sobre el todo si el tratamiento se realiza en centros que atienden elevados volúmenes de pacientes ${ }^{29}$.

La realización de la craneotomía también permite remover hematomas, la sangre y otras sustancias espasminógenas del espacio subaracnoideo, lo cual contribuye al control de la PIC y a evitar el desarrollo de hidrocefalia y vasoespasmo. Por otro lado, se logra la exclusión total y permanente del aneurisma roto en casi el $100 \%$ de los casos $^{42}$, lo que contrasta con aproximadamente un $20 \%$ de recanalizaciones que se registran con el tratamiento endovascular, hecho que aumenta el riesgo de resangramiento y la necesidad de reiteración de tratamientos invasivos. No obstante, no se trata de emular entre ambas opciones, lo mejor es tener la disponibilidad de ambas para indicar la más ventajosa, de acuerdo a las características de cada caso y momento en particular ${ }^{16,24}$

\section{Factores de mal pronóstico en cuan-} to a los resultados: Con la aplicación de técnicas de análisis estadístico multivariadas se comprobaron varios factores relacionados con la muerte y los malos resultados en esta serie. El resangramiento preoperatorio, sobre todo si produce o amplifica un hematoma intraparenquimatoso, es la complicación aguda de mayor mortalidad en los pacientes con HSAa y el hecho de ser de alto grado es uno de los factores de riesgo más importante para dicha complicación, cuya incidencia se ha aproximado a un $19 \%$ en varias series publicadas $^{33}$ Rivero-Rodríguez y colaboradores ${ }^{36}$ reportaron una mortalidad en estas condiciones del $61,7 \%$.

El edema cerebral constatado en el transoperatorio constituyó la variable más importante para predecir la mor- talidad en esta serie, determinado en el modelo de regresión logística. No se demostró desde el punto de vista estadístico asociación con la ocurrencia de ninguna complicación transopertatoria. En este caso podría plantearse la hipótesis de que fueron pacientes en los que habían ocurrido ya lesiones isquémicas del encéfalo no evidentes en la TC de cráneo, sin descartarse la posibilidad de falla en la autorregulación cerebral, hecho que como se sabe ensombrece el pronóstico de cualquier enfermo neurocrítico.

El valor más alto en la escala de Fisher se relacionó con la mortalidad e integró, junto con las complicaciones postoperatorias lideradas por el vasosespasmo sintomático, el modelo de mejor predicción para los malos resultados. Este hallazgo concuerda con los trabajos de Pereira y colaboradores ${ }^{30}$ y Zhao y colaboradores ${ }^{48,49}$, este último basado en el estudio multicéntrico de pacientes con HSAa de alto grado, identificado por el acrónimo AMPA, que involucró a 366 pacientes atendidos en varios hospitales Chinos entre los años 2010 al 2012.

Por último se detectó la asociación de la realización de ventriculostomía externa con la mortalidad y los malos resultados, hecho que a priori puede resultar contradictorio. Sin embargo, el análisis de varianza de la serie (ANOVA) aclara que dicho pronóstico se cumplió en los casos donde este procedimiento no se practicó previa a la cirugía para la oclusión del aneurisma, sino dentro de este propio acto o posterior al mismo, aspecto muy relevante y que nos lleva a la hipótesis de que la precocidad en la adopción de esta medida puede ser clave para obtener los resultados favorables que han sido bien demostrados en otras publicaciones y que fueron ya comentados en este trabajo.

En este estudio no se detectó ninguna variable relacionada con el acto quirúrgico es sí; como la ocurrencia de ruptura aneurismática transoperatoria, la aplicación de clipaje transitorio del vaso madre, ni la apertura o no de la lámina terminalis, causante de mortalidad o malos resultados, lo cual afirma que el grado de dificultad quirúrgica que muestra este grupo de enfermos no constituyó un factor de mal de pronóstico en esta serie.

Estrategias en el horizonte: A pesar de que los resultados con el trata- 
miento neuroquirúgico agresivo, que se inicia con la oclusión temprana del aneurisma roto para evitar el resangramiento, mejora de manera significativa la historia natural de la enfermedad en este subgrupo de enfermos con HSAa, los mismos son aun ominosos. Según se demostró en esta serie, concordante con otras ya comentadas en este trabajo, la mortalidad se aproxima al tercio de los pacientes operados y la autovalencia, al menos para satisfacer las necesidades personales, se logra en menos de la mitad de los casos. El hecho de que la disminución de la mortalidad se logre a expensas del aumento del número de pacientes con discapacidad severa, llama la atención sobre la necesidad de nuevas estrategias con mejores dividendos éticos y que al mismo tiempo permitan optimizar los recursos disponibles.

Los pacientes con HSAa de alto grado constituyen en realidad un grupo heterogéneo, con predominio en algunos del compromiso sólo funcional y en otros de lesiones estructurales del encéfalo, establecidas por alteraciones en el flujo sanguíneo regional de causa multifactorial, tales como: hipertensión endocraneana, pérdida de la autorregulación vascular, vasoespasmo ultratemprano o de la microcirculación, depresión eléctrica propagada y microtrombosis vascular, entre otros ${ }^{40}$.

En este sentido se han logrado avances en la selección de los pacientes que serán tratados mediante cirugía temprana. A pesar de que la evaluación neurológica partiendo de la suspensión transitoria de los fármacos sedantes, no ha demostrado un aumento en el riesgo de complicaciones y permite identificar a aquellos enfermos que mantienen ciertas funciones neurológicas, sobre todo motoras, esta puede subestimar la existencia de lesiones encefálicas relevantes, mediadas fundamentalmente por la isquemia, que ensombrecerán el pronóstico y por lo tanto hacen menos nítida la ventaja del tratamiento agresivo. Por otro lado, aunque está demostrada la evolución desfavorable en los enfermos que muestran lesiones isquémicas en la TC de cráneo, es bien conocida la baja sensibilidad de esta técnica para detectar dichas lesiones en el estadio agudo, hecho que obliga a la introducción de investigaciones más efectivas ${ }^{9}$.

Dentro de las técnicas que podrían ayudar para definir la existencia de lesión encefálica relevante resaltan en la literatura las siguientes: en el monitoreo con Doppler transcraneal la detección de velocidades en la arteria cerebral media mayores a $200 \mathrm{~cm} / \mathrm{segundo}$, una variabilidad entre exámenes mayor a $50 \mathrm{~cm} / \mathrm{segundo}$ e índice de Lindergard superior a 6 , se corresponden con reducción mayor al $70 \%$ de la luz arterial determinada en la Angio TC cerebral y se relaciona con vasoespasmo sintomático, el cual se asocia con malos resultados ${ }^{15,32}$.

La exploración con técnicas para determinar el flujo sanguíneo cerebral, tales como tomografía de emisión de positrones (PET), tomografía de emisión de fotón simple (SPECT), perfusión por RM y TC con Xenón estable (Xe/ $\mathrm{CT})$ son útiles para demostrar lesiones isquémicas desde la etapa aguda, sin embargo, algunas tienen las desventajas del alto costo, baja disponibilidad o largos periodos de adquisición de imágenes. En este sentido se ha mostrado las ventajas del estudio de la perfusión por TC, disponible en la mayoría de los centros y que requiere poco tiempo de realización; su sensibilidad para detectar lesiones isquémicas encefálicas es de casi el $100 \%$ cuando se registran flujos sanguíneos cerebrales regionales menores a $25 \mathrm{ml} / 100 \mathrm{~g} /$ minuto o tiempos circulatorios medios del encéfalo superiores a 6 segundos ${ }^{37,38}$.

La detección de la disminución de la variabilidad alfa en el monitoreo continuo con electroencefalograma, la caída de la presión tisular de oxígeno del encéfalo por debajo de $20 \mathrm{~mm} \mathrm{Hg}$, índice de Lactato/Piruvato superior a 40 o la detección de lesiones cerebrales extensas en la RM con técnica de difusión se han relacionado también con malos resultados ${ }^{39}$.

Aún se requieren de estudios con diseños robustos que prueben la utilidad de estas técnicas, sin embargo, se han publicado diferentes series que permiten evidenciar su probable utilidad para una adecuada selección de los enfermos con HSAa con pobre condición clínica, susceptibles a beneficiarse con la oclusión temprana del aneurisma roto, no solo para evitar la muerte sino también para lograr un resultado funcional aceptable para el individuo, sus familiares y la sociedad.

Limitaciones del estudio: Se trata de un estudio retrospectivo y trasversal de una serie pequeña de pacientes, lo que no garantiza la validación externa de los resultados obtenidos.

\section{Conclusiones}

La oclusión temprana de los aneurismas intracraneales rotos en pacientes con HSAa de alto grado es factible de lograrse. También es ventajosa para lograr disminuir de forma significativa la mortalidad en este grupo de enfermos. Sin embargo los resultados muestran aun un índice elevado de enfermos con discapacidad severa, hecho que evidencia la necesidad de profundizar en la aplicación de estrategias que permitan una mejor selección de los enfermos en los que es en realidad ventajoso adoptar esta actitud terapéutica.

Conflicto de intereses: No se declaran conflicto de intereses.

\section{Recibido: 25 de abril de 2017}

Aceptado: 30 de mayo de 2017

\section{Referencias}

1. Athar KM, Levine MJ. Treatment Options for Cerebral Vasospasm in Aneurysmal Subarachnoid Hemorrhage. Neurotherapeutics; 9: 3743. 2012. DOI: $10.1007 / \mathrm{s} 13311-011-0098-1$

2. Castanares-Zapatero $\mathrm{D}$, Hantson Ph. Pharmacological treatment of delayed cerebral ischemia and vasospasm in subaracnoid hemorrhage. Annals of Intensive Care: 12-20. 2011.

3. Castro AM. Understanding the Role of Hemodinamics in the Initiation, Progression, Rupture and Treatment Outcome of Cerebral Aneu- 
rysm from Medical Image-Based Computational Studies. ISRN Radiology. 2013 (citado el 3/2/2017) (aprox. 17 páginas). Disponible en: https://www.researchgate.net/publication/258405155. DOI: 10.5402/2013/602707.

4. Chalouhi N, Jabbour P, Dumont SA, González F, Rosenwasser R, Tjoumakaris IS. Endovascular Management of Subarachnoid Hemorrhage. En: Spetzler F R, Kalani M Y, Nakaji P, eds. Neurovascular Surgery. New York: Thieme Medical Publishers. 2015 ; p. $493-504$.

5. Chowdhury T, Capellani BR, Sandu N, Schaller B, Daya J. Perioperative Variables Contributieng to the Rupture of Intracranial Aneurysm: An Update. The Scientific World Journal. 2013 (citado el 3/2/2017) (aprox. 7 páginas). Disponible en: PubMed: 2013: 396404. DOI: $10.1155 / 2013 / 396404$.

6. Chua HM, Griessenauer JCh, Thomas JA, Ogilvy SCh. Who is Likely to Present in Poor Neurologic Condition After Aneurysmal Subaracnoid Hemorrhage? Risk Factors and Implications for Treatment. World Neurosurgery; 92: 113-119. 2016.

7. Cohen M, Jethwa P, Prestigiacomo JCh. Subarachnoid Hemorrhage: Workup and Diagnosis. En: Harbaugh ER, Shaffrey Ch, Couldwell TW, Berger SM, eds. Neurosurgery Knowledge Update. New York: Thieme Medical Publishers. 2015; p. 39-43.

8. Connolly SE, Rabinstein AA, Carhuapoma RJ, Derdeyn PC, Dion J, Hogashida TR, et al. Guidelines for the Management of Aneurysmal Subarachnoid Hemorrhage: A Guideline for Healthcare Professionals From the American Heart Association/American Stroke Association. Stroke; 43: 1711-1737. 2012.

9. de Oliveira M, Goffi A, Marotta RT, Schweizer AT, Abrahamson S, Macdonald LR. The critical care management of poor-grade subarachnoid haemorrhage. Critical Care; 20: 21-30. 2016. DOI: 10.1186/s13054-016-1193-9.

10. Dong WY, Young JJ, Byung YCh, Chul HCh. Subarachnoid Hemorrhage with Negative Baseline Digital Subtraction Angiography: Is Repeat Digital Subtraction Angiography Necessary?. J Cerebrovasc Neurosurg; 14(3): 210-215. 2012.

11. Ducruet FA, Gigante RP, Hickman LZ, Zaharia EB, Arias JE, Grobelny TB. Genetic determinants of cerebral vasospasm, delayed cerebral ischemia, and outcome after aneurysmal subarachnoid hemorrhage. Journal of Cerebral Blood Flow and Metabolism; 30: 676-688. 2010. DOI: $10.1038 / j \mathrm{jbfm} .2009 .278$.

12. Eleftherios A, Nazareno MN. Acute management of poor condition subarachnoid hemorrhage patients. Vascular Health and Risk Management; 3(6): 1075-82. 2007.

13. Ellis AJ, Connolly SE. Ruptured Intracranial Aneurysm: Indications for Microsurgery. En: Harbaugh ER, Shaffrey Ch, Couldwell TW, Berger SM, eds. Neurosurgery Knowledge Update. New York: Thieme Medical Publishers. 2015; p. 54-58.

14. Goel G, Gupta V, Chinchure S, Gupta A, Kaur G, Jha NA. A decade after International Subarachnoid Aneurysm Trial: Coiling as a first choice treatment in the management of intracranial aneurysms-Technical feasibility and early management outcomes. Asian $\mathrm{J}$ Neurosurg; 9(3): 137-43. 2014.

15. González-González LJ, Hernández-Zayas H, Bretón-Rosario LJ, Elizondo-Barriel L, López-Arbolay O, Salva-Camaño S, et al. Momento quirúrgico apoyado con Doppler transcraneal en pacientes con hemorragia subaracnoidea aneurismática. Rev. Chil. Neurocirugía; 41 : 59-70. 2015.

16. Grasso G, Perra G. Surgical management of ruptured small cerebral aneurysm: Outcome and surgical notes. Surg Neurol Int; 6: $185-190$. 2015. DOI: $10.4103 / 2152-7806.171257$.

17. Gupta G, Connolly S. Cerebral Vasospasm and Delayed Ischemic Complications Associatted with Subarachnoid Hemorrhage. En: Spetzler FR, Kalani MY, Nakaji P, eds. Neurovascular Surgery. New York: Thieme Medical Publishers. 2015; p. 478-83.

18. Hatefi M, Azhary S, Naebaghace H, Mohamandi RH, Jaafarpour M. The Effect of Fenestration of Lamina Terminalis on the Vasospasm and Shunt-Dependent Hydrocephalus in Patients Following Subarachnoid Haemorrhage. Journal of Clinical and Diagnostic research; 9(7): 15-18. 2015.

19. Hwang SJ, Hyun KM, Lee JH, Choi EJ, Kim HJ, Lee RN, et al. Endovascular coiling versus neurosurgical clipping in patients with unruptured intracranial aneurysm: a systematic review. BMC Neurology; 12: 99-105. 2012.

20. Ibrahim AAM, Ashmawy GAHO, Eassa AYE, Mansour OY. Hyperacute versus Subacute Coiling of Aneurysmal Sabarachnoid Hemorrhage a Short-term Outcome and Single Center Experience. Pilot Study. Front Neurol; 7: 79-85. 2016. DOI: 10.3389/fneur.2016.00079.

21. Kelliny M, Maeder Ph, Binaghi S, Levivier M, Regli L, Meuli R. Cerebral aneurysm exclusion by CT angiography based on subarachnoid hemorrhage pattern: a retrospective study. BMC Neurology; 11: 8-14. 2011.

22. Kobata $\mathrm{H}$, Sugie A, Yoritsure $\mathrm{E}$, Miyota $\mathrm{T}$, Toho $\mathrm{T}$. Intracranial extravasation of contrast médium during diagnostic $\mathrm{CT}$ angiography in the initial evaluation of subarachnoid hemorrhage: report of 16 cases and review of the literature. Springer Plus; 2: 413-22. 2013.

23. Koso M, Dizdarevic K, Sose SJ. Everyday Memory in Microsurgically Treated Patients After Subarachnoid Hemorrhage. J Clin Med Res; 7(4): 225-231. 2015.

24. Kósba GM, Czapiga B, Jarmundowicz W. Aneurismal subarachnoid hemorrhage: who remains for surgical treatment in the post-ISAT era?. Arch Med Sci; 11(3): 536-543. 2015. DOI: 10.5114/aoms.2013.37333.

25. Lanzino G, Rabinstein AA. Subarachnoid Hemorrhage. En: Spetzler FR, Kalani MY, Nakaji P, eds. Neurovascular Surgery. New York: Thieme Medical Publishers. 2015; p. 468-477.

26. Lehecka M, Frösen J, Korja M, Lehto H, Kivisaari R, Romani R, et al. Intracranial Aneurysms. En: Spetzler FR, Kalani MY, Nakaji P, eds. Neurovascular Surgery. New York: Thieme Medical Publishers. 2015; p. 457-467.

27. Oudshoorn CS, Rinkel EJG, Molyneux JA, Kerr SR, Mess DMS, Backes D, et al. Aneurysm Treatment $<24 \mathrm{~h}$ versus $24-72 \mathrm{~h}$ After Subarachnoid Hemorrhage. Neurocrit Care; 21: 4-13. 2014. DOI: 10.1007/s2028-014-9969-8.

28. Pan J, Zhan R, Wen L, Tong Y, Wan S, Zhon Y. Ultra-Early Surgery for Poor-Grade Intracranial Aneurysmal Subarachnoid Hemorrhage. A preliminary Study. Yosei Med J; 50(4): 521-24. 2009.

29. Pandey SA, Gemmete JJ, Wilson JT, Chaudhary N, Thompson GB, Morgenstern BL, Burke J. High Subarachnoid Hemorrhage Patient Volume Associated with Lower Mortality and Better Outcomes. Neurosurg; 77(3): 462-470. 2015. DOI: 10.1227/NEU.0000000000000850.

30. Pereira OMA, Silva PW, de Figueredo GE, Oliveira AH, Teixeira JM. Fisher revised scale for assessment of prognosis in patients with subarachnoid hemorrhage. Arq. Neuro-Psiquiatr; 69(6): 910-13. 2011. DOI: 10.1590/s0004-282x2011000700012.

31. Quian C, Yu X, Chen J, Gu Ch, Wang L, Chen L, et al. Effect of the drainage of cerebrospinal fluid in patients with aneurysmal subarachnoid hemorrhage. Medicine; 95: 41-48. 2016. DOI: 10.1097/MD.0000000000005140.

32. Quintana-Marín L. Vasoespasmo cerebral y déficit isquémico tardío en la hemorragia subaracnoidea aneurismática. Guías para su manejo clínico. Rev. Chil. Neurocirugia; 42: 168-173. 2016.

33. Quing SL, Ping Ch, Yuan XL, Zhang YL, Liang HY, Lin SD, et al. Systolic Blood Pressure Variability is a Nobel Risk Factor for Rebleeding in Acute Subarachnoid Hemorrhage. Medicine; 95(11): 1-6. 2016. DOI: 10,1097/MD.0000000000003028.

34. República de Chile, Ministerio de Salud 2007. Guía clínica Hemorragia Subaracnoidea secundaria a rotura de aneurismas cerebrales. V 2.2. 2013

35. Ringer JA. Cerebral Vasospasm. En: Harbaugh ER, Shaffrey Ch, Couldwell TW, Berger SM, eds. Neurosurgery Knowledge Update. New York: Thieme Medical Publishers. 2015; p. 49-53. 
36. Rivero-Rodríguez D, Scherle-Matamoros EC, Gutiérrez-Rojas RA, Pérez-Nellar J, Gómez-Viera N. Características clínicas y evolutivas asociados al resangrado en la hemorragia subaracnoidea aneurismática. Rev Cubana Neurol Neurocir; 4(2): 124-9. 2014.

37. Sanelli CP, Ugrec J, Johnson EC, Tan J, Segal ZA, Fink M, et al. Using quantitative CT perfusión for evaluation of delayed cerebral ischemia following aneurysmal suarachnoid hemorrhage. AJNR; 32(11): 2047-2053. 2011. DOI: 10.3174/ajnr.A2693.

38. Sasahara A, Suzuki K, Takahashi Y, Koseki H, Hirota K, Ohbuchi H, et al. Prognostic Assessment of Aneurysmal Subarachnoid Patients with WFNS Grade V by CT Perfusion on Arrival. World Neurosurgery; 92: 1-6. 2016. DOI: 10.1016/J.WNEU. 2016.04.097.

39. Sato K, Shimizu H, Fujimura M, Inoue T, Matsumoto $Y$, Tominaga T. Acute-stage diffusion-weighted magnetic resonance imaging for predicting outcome of por-grade aneurysmal subarachnoid hemorrhage. Journal of Cerebral Blood Flow and Metabolism; 30: 1110-1120. 2010. DOI: $10.1038 / j \mathrm{cbfm} .2009 .264$.

40. Sehba AF, Hou J, Pluta MR, Zhang HJ. The Importance of Early Brain Injury after Subarachnoid Hemorrhage. Prog Neurobiol; 97(1): 14-37. 2012. DOI: 10.1016/j.pneurobio.2012.02.003.

41. Sijercic AS, Brkic H, Audagic H, Surajic J, Hodzic S. Impact of Comorbidity on Early Outcome of Patients with Subarachnoid Hemorrhage Caused by Cerebral aneurysm Rupture. Med Arch; 69 (5): 280-283. 2015. DOI: 10.5455/medarch.2015.69.280-283.

42. Szmuda T, Sloniewski P. Early and long-term outcome of surgically treated giant internal carotid artery aneurysms-comparison with smaller aneurysms. Acta Neurochir; 153: 1611-1619. 2011. DOI: 10. 1007/s00701-011-1021-6.

43. Wright ES. Medical Management of Subarachnoid Hemorrhage. En: Spetzler FR, Kalani MY, Nakaji P, eds. Neurovascular Surgery. New York: Thieme Medical Publishers. 2015; p. 484-492.

44. Zang Y, Zhu X, Hou K, Zhao J, Gao X, Sun Y, et al. Clinical outcomes of surgical clipping for intracranial aneurysms in patients with Hunt and Hess grade 4 or 5. Arq.Neuro-Psiquiatr; 74(6): 478-81. 2016. DOI: 10.1590/0004-282x20160064.

45. Zeng S, Yao P, Yu L, Kang D. Keyhole Approach Combined With External Ventricular Drainage for Ruptured, Poor Grade, Anterior Circulation Cerebral Aneurysms. Medicine; 94(51): 1-8. 2015. DOI: 10.1097/MD.0000000000002307.

46. Zhang Q, Ma L, Liu Y, He M, Sung H, Wang X, et al. Timing of operation for poor-grade aneurysmal subaracnoid hemorrhage: study protocol for a randomized controlled trial. BMC Neurology; 13: 108-115. 2013.

47. Zhao B, Tan X, Yang H, Zhenk K, Li Z, Xion Y, et al. A Multicenter prospective study of poor-grade aneurysmal subarachnoid hemorrhage (AMPAS): observational registry study. BMC Neurology; 14: 86-92. 2014.

48. Zhao B, Yang H, Zheng K, Li Z, Xion Y, Tan X, et al. Predictors of good functional outcomes and mortality in patients with severe rebleeding after aneurysmal subarachnoid hemorrhage. Clinical Neurology and Neurosurgery; 144: 28-32. 2016. DOI: 10.1016/j.clineuro.2016.02.042.

49. Zhao B, Yang H, Zheng K, Li Z, Xion Y, Tan X, et al. Preoperative and postoperative predictors of long-term outcome after endovascular treatment of poor-grade aneurysmal subarachnoid hemorrhage. J Neurosurg; 1: 1-8. 2016.

\section{Correspondencia a:}

Dr. Ariel Varela Hernández

Servicio de Neurocirugía. Hospital Regional de Talca. Maule. Chile.

arelahernandezariel@gmail.com 